Original research article

\title{
Cognitive performance in young and middle-aged adults with migraine: Investigating the correlation with white matter hyperintensities and psychological symptoms
}

\author{
Abdulkadir Tunç ${ }^{a, *}$, Aysel Kaya Tekeşin ${ }^{b}$, Belma Doğan Güngen ${ }^{c}$, \\ Esra Arda ${ }^{d}$ \\ ${ }^{a}$ Clinic of Neurology, Bezmialem Vakıf University, İstanbul, Turkey \\ ${ }^{\mathrm{b}}$ Clinic of Neurology, Istanbul Education and Research Hospital, İstanbul, Turkey \\ c Clinic of Neurology, Reyap Hospital, Istanbul, Turkey \\ ${ }^{\mathrm{d} C l i n i c}$ of Psychiatry, Istanbul Education and Research Hospital, Istanbul, Turkey
}

\section{A R T I C L E I N F O}

\section{Article history:}

Received 31 October 2017

Accepted 2 May 2018

Available online 8 May 2018

Keywords:

Migraine

Cognitive performance

Depression

Anxiety

\begin{abstract}
A B S T R A C T
Introduction: This study aimed to evaluate the cognitive performance of migraine patients with $(\mathrm{MwA})$ and without aura (MwoA) and investigate the correlation of white matter hyperintensities (WMHs) and psychological symptoms with their cognitive test scores.

Material and methods: Hundred migraine patients aged $20-55$ years and 80 healthy volunteers with similar age, sex, and education level were enrolled. The total Montreal Cognitive Assessment (MoCA) scores were compared by age, sex, presence of aura, migraine duration, attack frequency, pain localization, presence and number of WMHs, and the scores of the Beck Depression Inventory and the Beck Anxiety Inventory (BAI).

Results: Forty-seven (47\%) patients had MwA and 53 (53\%) had MwoA. The performance of the MwA patients was significantly poorer than that of the MwoA patients and the healthy subjects on the MoCA scales. In particular, the results revealed lower scores in the subscales regarding visuospatial/executive functions, naming, memory, attention, and abstraction in MwA patients than in the MwoA patients. Compared to healthy controls, more number of migraine patients had WMHs. The presence and number of WMHs had no significant correlation with the MoCA scores of the migraine patients. There was a significant correlation of the BAI and BDI scores with the total MoCA scores considering all migraine patients. Conclusions: This study suggested that MwA may be associated with low cognitive performance which was correlated with depression and anxiety but not with WMHs. Further, longitudinal studies for assessing the relationship between WMHs, cognitive functions, and migraine, and for establishing the causality are warranted.
\end{abstract}

(c) 2018 Polish Neurological Society. Published by Elsevier Sp. z o.o. All rights reserved.

\footnotetext{
* Corresponding author at: Clinic of Neurology, Bezmialem University, Adnan Menderes Bulvarı (Vatan Cad.) Fatih, 34093 İstanbul, Turkey. E-mail addresses: drkadirtunc@hotmail.com (A. Tunç), atekesin@yahoo.com (A.K. Tekeşin), dr_belmadogan@hotmail.com (B.D. Güngen), esraarda@hotmail.com (E. Arda). https://doi.org/10.1016/j.pjnns.2018.05.001 0028-3843/@ 2018 Polish Neurological Society. Published by Elsevier Sp. z o.o. All rights reserved.
} 


\section{Introduction}

Migraine is one of the most common pain disorders, and the prevalence in the general population ranges from $5 \%$ to $20 \%$ [1]. It is characterized by recurrent throbbing headaches, nausea and/or vomiting, and arousal sensitivity to stimulants such as light, sound, and movement. A significant proportion of the population (up to $21 \%$ of women and $6 \%$ of men) experiences migraine attacks $[1,2]$. As the headache progresses, various autonomic, affective, cognitive, and sensory symptoms may occur [3]. The extent of these different symptoms suggests that migraine is more than a headache. It is a complex neurological disorder affecting multiple cortical, subcortical, and brainstem regions; therefore, it is clear that the migraine brain differs from the non-migraine brain [4].

Increased evidence suggests that in many individuals, migraine is a chronic disease associated with significant comorbidities, including white matter hyperintensities (WMHs), which are associated with volume changes in the white and gray matter regions of the brain [5]. The pathophysiology of WMHs is unknown. Cortical spreading depression (CSD) is defined as a propagating wave of glial and neuronal depolarization that occurs during migraine aura [6]. CSD results in partial activation of matrix metalloproteinases, hypoperfusion of small penetrating arteries, and altered vascular permeability. This may cause hypoxic brain injury, manifesting as WMHs on magnetic resonance imaging (MRI) [6]. Atherosclerosis and increased age may be the main risk factors for the development of WMHs but in migraine patients, attack frequency, the duration of disease, presence of aura and possible comorbid disease are also important in the development of WMHs [7,8]. Stroke and WMHs are associated with an increased risk of dementia [9]; therefore, it can be hypothesized that migraine patients may have impaired cognitive function. Some previous studies have shown a deleterious effect of migraine on many cognitive skills, including verbal ability, attention, psychomotor ability, and memory $[10,11]$. Especially presence of migraine-related aura has been shown to be associated with cognitive impairment $[12,13]$. However, other studies have not shown any effect of migraine on cognitive skills $[14,15]$. Discrepancies between these studies may possibly be attributed to insufficient neuropsychological assessments. Moreover, to our knowledge, only a few studies have considered the possible relationship between cognitive performance and the psychological symptoms or behavioral disturbances frequently seen in migraine patients $[4,16]$.

This study aimed to evaluate the cognitive status of migraine patients with and without aura and investigate the association of WMHs and psychological symptoms with their cognitive function.

\section{Material and methods}

This prospective case-control study was performed at the Neurology Clinic between May 2016 and May 2017. Hundred migraine patients aged $20-55$ years and 80 healthy volunteers with similar age, sex, and education level were enrolled.

The study was approved by local ethics committee. A detailed, written informed consent form was obtained from each subject before initiating the study. The inclusion criterion for migraine patients was the diagnosis of migraine according to the International Classification of Headache Disorders III (beta version) (ICHD-III) [17]. Exclusion criteria were as follows: other ICHD-III diagnosis (e.g., tension type headache, cluster headache, etc.); history of cerebrovascular disease, cardiovascular disease, diabetes mellitus, arterial hypertension, or hyperlipidemia; somatic or psychiatric disorders (e.g., major depression or psychosis according to DSM-5 criteria); smoking cigarettes or alcohol/substance abuse; current pregnancy, lactation, or hormonal contraceptive use; use of drugs such as antiplatelet agents, anticoagulants, statins, or hormonal drugs; renal, metabolic, inflammatory, infectious, or immune disease; or possible "symptomatic migraine" in which the MRI showed disorders such as ischemic infarcts, arteriovenous malformations, or brain tumors.

For at least 3 days before and after the neuropsychological tests, all the patients were migraine free and were not taking rescue medications to avoid any possible impact related to the attacks or to the pharmacological treatments. To ensure this, all patients were interviewed 3 days after the cognitive assessments.

The control group patients were selected from among the relatives of the migraine patients, hospital employees, or the general population. Written consent was obtained from them for voluntary participation in the study. The inclusion criteria for the control group were as follows: absence of migraine, the maximum frequency of any headache less than 1 episode per month (e.g., tension type headache, or any other type of chronic headache) and the absence of a diagnosed psychiatric disorder (e.g., major depression or psychosis). Exclusion criteria for the healthy controls were the same as those for the migraine patients.

Demographic and clinical characteristics such as disease duration, frequency of migraine attacks (number of migraine attacks per month), pain localization, and the presence of aura were recorded.

\subsection{Cognitive assessment}

The Montreal Cognitive Assessment (MoCA) [18] was used for evaluating the cognitive function of the migraine patients and healthy controls. The MoCA is a 30-point test that assesses several cognitive domains; it takes $10-15 \mathrm{~min}$ to complete. Mild CI is indicated by a total score $<15.5$ on the following subscales: visuospatial/executive functions, naming, memory, attention, language, abstraction, and orientation [19].

\subsection{Assessment of depression and anxiety}

Depression and anxiety symptoms were evaluated using the BDI and the BAI scores, respectively. The Beck depression and anxiety inventories comprise 21 questions and are scored between 0 and 63 points. The depression and anxiety cut-off values were taken as BDI score $\geq 10$ points and BAI score $\geq 17$ points, respectively $[20,21]$.

\subsection{White matter hyperintensities}

All participants underwent whole-brain MRI using the same 1.5 T MRI scanner (Siemens Verio). The scans included $\geq 3$ 
sequences: axial T2-weighted, axial fluid attenuated inversion recovery (FLAIR), and sagittal T1-weighted images. The slice thickness was $5 \mathrm{~mm}$, the gap was $1 \mathrm{~mm}$, and no intravenous contrast was used.

All MRI scans were reviewed and scored by the same neuroradiologist who was blinded to the clinical details. The scans were visually assessed for the presence and features of WMHs including appearance, number, and distribution. WMHs were defined as clearly hyperintense areas relative to the surrounding white matter on both, FLAIR and $\mathrm{T}_{2}$-weighted images and identified by simultaneous inspection of both aligned images.

The number of WMHs was determined on the FLAIR images and grouped according to their location and distribution. Subgroups were delineated according to the distribution of the WMHs according to the methodology previously described for multiple sclerosis patients [22]. These subgroups were subcortical, juxtacortical, and periventricular. The locations of the WMHs were defined as frontal, parietal, temporal, occipital, or infratentorial. We included focal and punctate hyperintensities (size $<9 \mathrm{~mm}$ ) and excluded confluent and large hyperintensities (size $>9 \mathrm{~mm}$ ).

Migraine patients who had WMHs that were detected on brain MRI were evaluated using laboratory tests for vasculitis (antinuclear antibody, lupus anticoagulant, anticardiolipin antibodies, antidouble-stranded DNA autoantibody, as well as C3 and C4 levels). They also underwent cardiac examination to rule out patent foramen ovale and atrial septal defect.

\subsection{Statistical analysis}

The data were transferred into the IBM SPSS Statistics 23 Program and the analyses were completed. When descriptive statistics $(N, \%)$ were used for categorical variables, the descriptive statistics (mean, standard deviation) were given for numerical variables. Independent $t$ test was used to examine the difference between the categorical variables having two groups; whereas, one-way ANOVA test (one way analysis of variance) was used for examining the difference between the categorical variables having more than two groups. While chi square test was used for examining the correlation between the two categorical variables, Pearson Correlation Coefficients were used for examining the correlation between the two numerical variables. In order to investigate the effect of the independent variables on this dependent variable, the multiple linear regressions was used.

\section{Results}

Four patients were excluded since they were experienced migraine attacks during the 3 days following the cognitive assessments. Demographic and clinical characteristics, cognitive status, as well as depression and anxiety scores of the patient and control groups are shown in Table 1. No significant difference was detected between the groups in terms of age, sex, and formal education level $(p=0.4, p=0.216$, and $p=0.160$, respectively).

In the migraine group, 29 patients (29\%) were primary school graduates, 12 (12\%) were secondary school graduates,
31 were (31\%) high-school graduates, and 28 (28\%) were university graduates. In the control group, 12 individuals (15\%) were primary school graduates, 16 (20\%) were secondary school graduates, 30 (37\%) were high-school graduates, and 22 (27.5\%) were university graduates. The mean education levels of the migraine patients and healthy subjects were $10 \pm 3.9$ and $10.7 \pm 3.6$ years, respectively. There was also no difference between the education levels of MwA and MwoA patients (9.8 \pm 4 and $10.3 \pm 3.5$ years, respectively, $p$ : 0.230).

Forty-seven (47\%) patients had migraine with aura (MwA) and 53 (53\%) had migraine without aura (MwoA). Thirty-two patients reported headache in the right half of the head, while 24 patients had a headache in the left half of the head. Fortyfour patients reported bilateral, generalized throbbing pain.

\subsection{Cognitive assessment}

No significant difference was found in the total MoCA scores of the migraine patients and healthy subjects $(p=0.138)$. Four migraine patients were found to have mild CI as per the MoCA scale (score $<15.5$ ) (Table 1$)$.

The cognitive performance of the MwA patients was significantly poorer than that of the MwoA patients and the healthy subjects ( $p=0.014$ and $p=0.030$, respectively). The mean MoCA scores of the MwA patients, MwoA patients, and healthy controls were $21.7 \pm 5,24.9 \pm 3.5$, and $24.4 \pm 3.8$, respectively. No significant relationship was found between the total MoCA scores of the MwoA patients and the healthy controls $(p=0.547)$.

When we evaluated the MoCA subdomains using linear regression analysis, presence of aura was found to be associated with visuospatial/executive functions, naming, memory, attention, and abstraction domains in migraine patients $(p=0.003, p=0.044, p=0.028, p=0.001$, and $p=0.001$, respectively). When we compared the 3 groups (MwA, MwoA and the control group) with one-way ANOVA test, the total MOCA scores of the MwA group was statistically significantly lower $(p<0.05)$.

\subsection{Analysis of the association between depression, anxiety and cognitive function}

No significant difference was detected between the migraine patients and healthy controls in terms of their BDI and BAI scores $(p=0.719, p=0.836)$ (Table 1$)$. There was no significant correlation of the BDI scores with the total MoCA scores when all participants were considered $(r:-145, p=0.052)$. On the contrary, a statistically significant correlation was found between the BDI and total MoCA scores in migraine patients $(p=0.042)$. The BDI scores were significantly correlated to memory, attention, and abstraction domains $(p=0.002$, $p=0.041$, and $p=0.016$, respectively).

We found a significant correlation of the BAI score with the total MoCA scores in migraine patients $(r:-0.248, p=0.013)$. Cognitive performance pertaining to memory, attention, language, and abstraction was found to be correlated with the BAI scores $(p=0.001, p=0.018, p=0.002$, and $p=0.001$, respectively). The correlations of the BDI and BAI scores with the total MoCA scores and the MoCA subdomains in migraine patients are shown in Table 2. 
Table 1 - Demographic and clinical characteristics, cognition, depression and anxiety scores of the patient and the control groups.

\begin{tabular}{|c|c|c|c|}
\hline Gender $(\mathrm{m} / \mathrm{f}) n(\%)$ & $\begin{array}{c}\text { Migraine group (n:100) } \\
\text { 9/91 (9/91) } \\
\text { Mean } \pm \text { SD }\end{array}$ & $\begin{array}{c}\text { Control group (n:80) } \\
\text { 10/70 (12.5/87.5) } \\
\text { Mean } \pm \text { SD }\end{array}$ & $\begin{array}{l}p^{*} \\
0.4\end{array}$ \\
\hline Migraine duration (year) & $7.4 \pm 7.1$ & - & \\
\hline Attack frequency (per month) & $3.6 \pm 2.09$ & - & \\
\hline Presence of aura $n(\%)$ & $47(47)$ & - & \\
\hline Age (year) & $36.7 \pm 9.4$ & $34.4 \pm 11.02$ & 0.216 \\
\hline BAI score & $17.5 \pm 12.1$ & $17.1 \pm 11.1$ & 0.836 \\
\hline BAI $(\geq 17) n(\%)$ & $43(43)$ & $31(38.8)$ & 0.565 \\
\hline BDI score & $15.1 \pm 10.3$ & $15.3 \pm 9.2$ & 0.719 \\
\hline $\mathrm{BDI}(\geq 10) n(\%)$ & $60(60)$ & $51(63.8)$ & 0.607 \\
\hline MoCA score & $23.4 \pm 4.5$ & $24.4 \pm 3.8$ & 0.138 \\
\hline MoCA $(<15.5) n(\%)$ & $4(4)$ & $0(0)$ & 0.130 \\
\hline Presence of WMHs $n(\%)$ & $32(32)$ & $15(18.8)$ & 0.044 \\
\hline Number of WMHs & $3.5 \pm 4.7$ & $2.5 \pm 1.5$ & 0.353 \\
\hline Visuospatial score & $4.08 \pm 1.02$ & $4.1 \pm 0.906$ & 0.568 \\
\hline Naming score & $2.64 \pm 0.5$ & $2.74 \pm 0.522$ & 0.181 \\
\hline Memory score & $2.4 \pm 1.5$ & $2.5 \pm 1.6$ & 0.595 \\
\hline Attention score & $4.6 \pm 1.3$ & $4.9 \pm 1.2$ & 0.167 \\
\hline Language score & $2.05 \pm 1.03$ & $2.3 \pm 0.9$ & 0.075 \\
\hline Abstraction score & $1.6 \pm 0.59$ & $1.9 \pm 0.38$ & 0.032 \\
\hline Orientation score & $5.97 \pm 0.171$ & $5.96 \pm 0.191$ & 0.783 \\
\hline
\end{tabular}

Table 2 - The association between the MoCA subdomains, patient characteristics, and other evaluated data in migraine patients.

\begin{tabular}{|c|c|c|c|c|c|c|c|c|}
\hline$\left(p^{*}\right)$ & $\begin{array}{l}\text { Visuospatial } \\
\text { functions }\end{array}$ & Naming & Memory & Attention & Language & Orientation & Abstraction & $\begin{array}{c}\text { Total } \\
\text { MoCA scores }\end{array}$ \\
\hline \multirow[t]{2}{*}{ Age } & $p: 0.007$ & $p: 0.248$ & $p: 0.001$ & $p: 0.004$ & $p: 0.036$ & $p: 0.585$ & $p: 0.01$ & $p: 0.001$ \\
\hline & $r:-0.267$ & $r:-0.117$ & $r:-0.378$ & $r:-0.285$ & $r:-0.210$ & $r: 0.055$ & $r: 0.289$ & $r:-.363$ \\
\hline \multirow[t]{2}{*}{ Education level } & $p: 0.001$ & $p: 0.001$ & $p: 0.01$ & $p: 0.01$ & $p: 0.001$ & $p: 0.898$ & $p: 0.001$ & $p: 0.001$ \\
\hline & $r: 0.557$ & $r: 0.368$ & $r: 0.578$ & $r: 0.505$ & $r: 0.449$ & $r: 0.013$ & $r: 0.488$ & $r: 0.677$ \\
\hline \multirow[t]{2}{*}{ Pain localization } & $p: 0.362$ & $p: 0.451$ & $p: 0.558$ & $p: 0.022$ & $p: 0.03$ & $p: 0.809$ & $p: 0.44$ & $p: 0.121$ \\
\hline & $r: 0.092$ & $r: 0.076$ & $r:-0.059$ & $r:-0.230$ & $r:-0.218$ & $r: 0.024$ & $r:-0.07$ & $r: 0.273$ \\
\hline \multirow[t]{2}{*}{ Attack frequency } & $p: 0.023$ & p: 0.094 & $p: 0.046$ & $p: 0.075$ & $p: 0.002$ & $p: 0.770$ & $p: 0.002$ & $p: 0.001$ \\
\hline & $r: 0.293$ & $r: 0.168$ & $r:-0.200$ & $r:-0.179$ & $r:-0.310$ & $r:-0.03$ & $r:-0.310$ & $r:-.304$ \\
\hline \multirow[t]{2}{*}{ Migraine duration } & $p: 0.139$ & $p: 0.469$ & $p: 0.385$ & $p: 0.653$ & $p: 0.185$ & $p: 0.177$ & $p: 0.032$ & $p: 0.165$ \\
\hline & $r: 0.149$ & $r: 0.073$ & $r: 0.088$ & $r:-0.045$ & $r:-0.134$ & $r:-0.136$ & $r:-0.214$ & $r:-.140$ \\
\hline \multirow[t]{2}{*}{ BAI } & $p: 0.313$ & $p: 0.135$ & p: 0.001 & $p: 0.018$ & $p: 0.002$ & $p: 0.427$ & $p: 0.001$ & $p: 0.013$ \\
\hline & $r: 0.01$ & $r: 0.151$ & $r:-0.342$ & $r:-0.236$ & $r:-0.305$ & $r: 0.08$ & $r:-0.315$ & $r:-.248$ \\
\hline \multirow[t]{2}{*}{ BDI } & $p: 0.152$ & $p: 0.382$ & $p: 0.002$ & $p: 0.041$ & $p: 0.096$ & $p: 0.083$ & $p: 0.016$ & $p: 0.042$ \\
\hline & $r: 0.144$ & $r: 0.088$ & $r:-0.306$ & $r:-0.205$ & $r:-0.167$ & $r:-0.015$ & $r:-0.238$ & $r:-.280$ \\
\hline \multirow[t]{2}{*}{ Number of WMHs } & $p: 0.810$ & $p: 0.437$ & $p: 0.934$ & $p: 0.361$ & $p: 0.501$ & $p: 0.862$ & $p: 0.810$ & $p: 0.597$ \\
\hline & $r:-0.04$ & $r:-0.130$ & $r: 0.014$ & $r:-0.153$ & $r:-0.113$ & $r: 0.029$ & $r:-0.04$ & $r:-.089$ \\
\hline
\end{tabular}

\subsection{White matter hyperintensities and cognition}

Compared to healthy controls, a significantly higher number of migraine patients had WMHs $(p=0.044)$ (Table 1$)$. No significant relationship was found in terms of the number of WMHs per subject ( $p=0.353)$. WMH was detected in $12(25.5 \%)$ of the MwA patients and in 20 (37.7\%) of the MwoA patients. The WMHs in the migraine and control groups were detected most frequently in the frontal lobe and least frequently in the occipital lobe. In the 100 migraine patients, we found periventricular WMHs in $2(2 \%)$, juxtacortical WMHs in 7 (7\%: frontal $4 \%$, parietal $1 \%$, frontal and parietal $2 \%$ ), and subcortical WMHs in 32 patients (32\%: frontal 13\%, parietal 3\%, temporal $2 \%$, occipital $1 \%$, multiple localization $13 \%$ ). The presence and number of WMHs was not significantly correlated with the total MoCA scores nor with the MoCA sub-domains of the migraine patients (Table 2). There was no significant difference between WMHs (+) and WMHs (-) migraine patients in terms of attack frequency, disease duration and total MoCA scores ( $p=0.683, p=0.420$, and $p=0.204$, respectively). 


\subsection{Cognitive impairment and other parameters}

We evaluated the association of total MoCA scores with other parameters in migraine patients as one group (without differentiation between those with and without aura) to maintain the statistical clarity of the study.

Attack frequency was significantly correlated to the MoCA scores in migraine patients but migraine duration was not (Table 2). Additionally, pain localization was not correlated to the total MoCA scores in MwA patients ( $p$ : 0.095).

Finally, we evaluated the MoCA subdomains, patient characteristics, and other evaluated data using linear regression analysis in migraine patients. BAI was associated with memory, attention, language and orientation domains ( $p=0.05, p=0.011, p=0.032$ and $p=0.001$, respectively) and BDI was only associated with orientation $(p=0.018)$. When the total MoCA scores were evaluated with linear regression analysis, no significant differences were detected with the patient characteristics, and other evaluated data $(p>0.05)$.

\section{Discussion}

The present study revealed that MwA patients had significantly lower total MoCA scores than MwoA patients and healthy controls. In particular, our results revealed lower scores in the subscales regarding visuospatial/executive functions, naming, memory, attention, and abstraction in MwA patients than in the MwoA patients. Regarding the psychological assessment, the migraine patients and healthy subjects reported similar scores for depression (BDI) and anxiety (BAI). In patients with migraine, a statistically significant correlation was found between the BDI and BAI scores and total MoCA scores. Compared to healthy controls, more number of migraine patients had WMHs. Cognitive scores in migraine patients were not associated with the presence and number of WMHs.

The prevalence of WMHs in migraine patients ranges from $14 \%$ to $59 \%$ [8]. Although the mechanisms and causes of WMHs are still debatable and many theories have been proposed, clear data showing the pathophysiological correlations are still lacking. Some peptides (e.g., substance $\mathrm{P}$, calcitonin generelated peptide, and neurokinin A) are released from the perivascular trigeminal regions after the activation of the brain tissue; this causes dilation and inflammation of the extraparenchymal vessels. This could lead to cerebral ischemia caused by the effects on the cerebral blood flow [6]. On the contrary, inflammatory arteriopathy of the cranial vessels is associated with migraine attacks [23].

A recent meta-analysis showed an association between WMHs and MwA; however, this association was not seen in MwoA patients [5]. Another meta-analysis, carried out by Schurks et al. [24], included 25 heterogeneous studies and showed that the WMH burden was higher in migraine patients and was correlated to each type of migraine, with the MwA patients having the highest number of WMHs. In keeping with previous findings, the presence of WMHs was significantly higher in migraine patients than in the healthy controls in our study. The WMH burden in MwoA patients was greater than that in MwA patients. Several issues regarding these WMHs, including whether they accumulate over time in repeat MRI assessments, whether hyperintensities constitute a risk for stroke, and whether they have an impact on cognitive performance in migraine patients, remain debatable. Migraine-related WMHs need to be distinguished from white matter lesions that are related to cerebral small-vessel disease and silent brain infarcts, both of which are associated with a risk of future stroke, cognitive decline, and dementia [25]. Therefore, we excluded confluent and large hyperintensities similar to a previous study [23].

Studies on the relationship between migraine and cognitive performance show contradictory results. Some cross-sectional studies have not found any differences between migraine patients and healthy controls [15,16], while others have shown evidence of cognitive decline among migraine patients compared to that in controls $[12,26]$. Interestingly, some studies have reported that migraineurs have shown less cognitive decline compared to nonmigraineurs [27,28]. Discrepancies between these studies may possibly be attributed to the differences in patient characteristics (some studies enrolled both, patients with and without aura), number of patients, or the differences in the neuropsychological assessments performed. In our study, no significant differences were found between the results of the cognitive abilities of the migraine patients and healthy controls. The MwA patients had significantly lower total MoCA scores than healthy subjects, while the cognitive performance of the MwoA patients and healthy controls was found to be comparable. In particular, in consensus with the findings of previous studies, we found that in comparison to the MwoA patients, the MwA patients achieved lower scores in the subscales assessing visuospatial/ executive functions, naming, verbal memory, attention, and abstraction $[12,13,29]$. These findings may be compatible with the hypothesis that subtle alterations in the information processing mechanisms could be present in the early stages of migraine [13]. The decline in verbal memory might be attributed to the defective strategic and organizational aspects of learning [29], also in consideration of the lower executive functions and attention subdomains.

In our study, the presence and number of WMHs were not correlated to cognitive functioning in migraine patients. Similarly, in the EVA study that used the MRI data of 775 participants, the presence of WMHs was not found to be associated with the development of migraine and cognitive deficits using most cognitive tests [30].

Previous studies assessed the cognitive functions of migraine patients and found that the attack frequency and disease duration were not related to cognitive decline [13,31]. We found a significant relationship between the attack frequency, migraine duration, and MoCA scores, which may be attributed to the different sample sizes or cognitive scales.

Regarding the psychological assessment, there were no significant differences in the severity of depression and anxiety in migraine patients compared to that in healthy subjects. We found a statistically significant correlation between depression, anxiety, and total MoCA scores in migraine patients. Both, depression and anxiety have been shown to be early predictors of future cognitive decline in previous studies $[32,33]$. However, another study has reported that anxiety and depression in elderly people is not associated 
with an increased risk of dementia or cognitive decline [34]. A study including MwoA patients revealed no correlation between neuropsychiatric symptoms and cognitive tests [1]. The divergence among the studies might be due to the difference in the sample sizes, inclusion and exclusion criteria, and tools used for assessing the neuropsychiatric profile and cognition.

We aimed to evaluate the general cognitive functioning of migraine patients, for which we used a relatively recent tool (the MoCA test). The MoCA has been developed for assessing several cognitive subdomains that are often overlooked by more common screening tools. Nevertheless, our study requires a complete and specific battery of anyway, especially in relation to the various subdomains and neuropsychiatric symptoms such as anxiety and depression. The cut off score derived for the MoCA test was consistent with Italian norms in this study [19]. However, some research has suggested that a higher normative data would yield more accurate results [35]. All subjects were scanned using the same MRI scanner at a single center. However, an MRI scanner with a higher magnetic field and higher sensitivity than the one used in our study would have been more sensitive in detecting the WMHs. The sample size was small, especially, the number of patients with WMHs, as determined using MRI was lower than required for drawing meaningful and representative results. Assessment of WMHs with validated methods would be more convenient (e.g., Fazekas score, Scheltens' score [36,37]). Additionally, contrary to the previous studies [38,39], a higher number of patients had MwA in our study and we did not evaluate the subtypes of MwA. More accurate results can be obtained with a larger sample size and longer follow-up periods. Finally, our study was cross-sectional indicating an association rather than a causal relationship. We did not evaluate the changes in the cognitive functions over time although dementia is usually determined by observing these changes. Longitudinal studies are needed in future to better understand the mechanisms of cognitive deficits in this population.

\section{Conclusions}

This study suggested that the effects of migraine are not limited to headaches. Especially migraine with aura may be associated with some comorbidities, including cognitive decline. Whether migraine patients with WMHs are at greater risk of cognitive decline than those without WMHs is under debate and needs to be prospectively evaluated. Thus, these results also highlighted the importance of carefully assessing the cognitive performance using a screening tool that is as sensitive as the MoCA test in detecting visuospatial/executive, attention, and memory dysfunctions. Further, longitudinal studies for assessing the relationship between WMHs, cognitive decline, and migraine, and for establishing the causality are warranted.

\section{Authors' contributions}

AT, AKT and BDG contributed to the study design and concept. AT, BDG and EA contributed to analysis and interpretation of data. AT and AKT were responsible for primary data analysis. AT, AKT, BDG and EA contributed to the writing and review. All authors read and approved the final manuscript.

\section{Conflict of interest}

None declared.

\section{Acknowledgement and financial support}

None declared.

\section{RE F E R E N C E S}

[1] Lipton RB, Bigal ME. Migraine: epidemiology, impact, and risk factors for progression. Headache 2005;45:3-13.

[2] Ferrante T, Castellini P, Abrignani G, Latte L, Russo M, Camarda C, et al. The PACE study: past-year prevalence of migraine in Parma's adult general population. Cephalalgia 2012;32:358-6510.

[3] Kelman L, Tanis D. The relationship between migraine pain and other associated symptoms. Cephalalgia 2006;26:548-53.

[4] Burstein R, Noseda R, Borsook D. Migraine: multiple processes, complex pathophysiology. J Neurosci 2015;35:6619-29.

[5] Bashir A, Lipton RB, Ashina S, Ashina M. Migraine and structural changes in the brain: a systematic review and meta-analysis. Neurology 2013;81:1260-8.

[6] Sinclair AJ, Matharu M. Migraine, cerebrovascular disease and the metabolic syndrome. Ann Indian Acad Neurol 2012;15:72-7.

[7] Kurth T, Mohamed S, Maillard P, Zhu YC, Chabriat H, Mazoyer B, et al. Headache, migraine, and structural brain lesions and function: population based epidemiology of vascular ageing-MRI study. BMJ 2011;342:c7357

[8] Seneviratne U, Chong W, Billimoria PH. Brain white matter hyperintensities in migraine: clinical and radiological correlates. Clin Neurol Neurosurg 2013;115:1040-3.

[9] Vermeer SE, Prins ND, den Heijer T, Hofman A, Koudstaal PJ, Breteler MM. Silent brain infarcts and the risk of dementia and cognitive decline. N Engl J Med 2003;348:1215-22.

[10] Camarda C, Monastero R, Pipia C, Recca D, Camarda R. Interictal executive dysfunction in migraineurs without aura: relationship with duration and intensity of attacks. Cephalalgia 2007;27:1094-100.

[11] Martins IP, Gil-Gouveia R, Silva C, Maruta C, Oliveira AG. Migraine, headaches, and cognition. Headache 2012;52:1471-82.

[12] Le Pira F, Zappala G, Giuffrida S, Lo Bartolo ML, Reggio E, Morana $\mathrm{R}$, et al. Memory disturbances in migraine with and without aura: a strategy problem? Cephalalgia 2000;20:475-8.

[13] Le Pira F, Lanaia F, Zappalà G, Morana R, Panetta MR, Reggio E, et al. Relationship between clinical variables and cognitive performances in migraineurs with and without aura. Funct Neurol 2004;19:101-5.

[14] Gaist D, Pedersen L, Madsen C, Tsiropoulos I, Bak S, Sindrup $\mathrm{S}$, et al. Long-term effects of migraine on cognitive function: a population-based study of Danish twins. Neurology 2005;64:600-7.

[15] Pearson AJ, Chronicle EP, Maylor EA, Bruce LA. Cognitive function is not impaired in people with along history of migraine: a blinded study. Cephalalgia 2006;26:74-80. 
[16] Santangelo G, Russo A, Trojano L, Falco F, Marcuccio L, Siciliano M, et al. Cognitive dysfunctions and psychological symptoms in migraine without aura: a cross-sectional study. J Headache Pain 2016;17:76.

[17] Headache Classification Subcommittee of the International Headache Society. The international classification of headache disorders: 3rd edition (beta version). Cephalalgia 2013;33:629-808.

[18] Smith T, Gildeh N, Holmes C. The Montreal Cognitive Assessment: validity and utility in a memory clinic setting. Can J Psychiatry 2007;52:329-32.

[19] Santangelo G, Siciliano M, Pedone R, Vitale C, Falco F, Bisogno R, et al. Normative data for the Montreal Cognitive Assessment in an Italian population sample. Neurol Sci 2015;36(4):585-91.

[20] Beck AT, Epstein N, Brown G, Steer RA. An inventory for measuring clinical anxiety: psychometric properties. J Consult Clin Psychol 1988;56:893-7.

[21] Beck AT, Steer RA. Internal consistencies of the original and revised Beck Depression Inventory. J Clin Psychol 1984;40:1365-7.

[22] Barkhof F, Filippi M, Miller DH, Scheltens P, Campi A, Polman $\mathrm{CH}$, et al. Comparison of MRI criteria at first presentation to predict conversion to clinically definite multiple sclerosis. Brain 1997;120:2059-69.

[23] Avci AY, Lakadamyali H, Arikan S, Benli US, Kilinc M. High sensitivity C-reactive protein and cerebral white matter hyperintensities on magnetic resonance imaging in migraine patients. J Headache Pain 2015;16:9.

[24] Schurks M, Rist PM, Bigal ME, Buring JE, Lipton RB, Kurth T. Migraine and cardiovascular disease: systematic review and meta-analysis. BMJ 2009;339:b3914.

[25] Paemeleire K. Brain lesions and cerebral functional impairment in migraine patients. J Neurol Sci 2009;283:134-6.

[26] Calandre EP, Bembibre J, Arnedo ML, Becerra D. Cognitive disturbances and regional cerebral blood flow abnormalities in migraine patients: their relationship with the clinical manifestations of the illness. Cephalalgia 2002;22:291-302.

[27] Wen K, Nguyen NT, Hofman A, Ikram MA, Franco OH. Migraine is associated with better cognition in the middleaged and elderly: the Rotterdam Study. Eur J Neurol 2016;23:1510-6.
[28] Kalaydjian A, Zandi PP, Swartz KL, Eaton WW, Lyketsos C. How migraines impact cognitive function: findings from the Baltimore ECA. Neurology 2007;68:1417-24.

[29] Schmitz N, Arkink EB, Mulder M, Rubia K, Admiraal-Behloul F, Schoonman GG, et al. Frontal lobe structure and executive function in migraine patients. Neurosci Lett 2008;440:92-6.

[30] Rist PM, Dufouil C, Glymour MM, Tzourio C, Kurth T. Migraine and cognitive decline in the population-based EVA study. Cephalalgia 2011;31:1291-300.

[31] Mulder EJ, Linssen WH, Passchier J, Orlebeke JF, de Geus EJ. Interictal and postictal cognitive changes in migraine. Cephalalgia 1999;19:557-65.

[32] Sinoff G, Werner P. Anxiety disorder and accompanying subjective memory loss in the elderly as a predictor of future cognitive decline. Int J Geriatr Psychiatry 2003;18:951-9.

[33] Banks SJ, Raman R, He F, Salmon DP, Ferris S, Aisen P, et al. The Alzheimer's Disease Cooperative Study Prevention Instrument Project: longitudinal outcome of behavioral measures as predictors of cognitive decline. Dement Geriatr Cogn Dis Extra 2014;4:509-16.

[34] Bierman EJM, Comijs HC, Jonker C, Scheltens P, Beekman ATF. The effect of anxiety and depression on decline of memory function in Alzheimer's disease. Int Psychogeriatr 2009;21:1142-7.

[35] Nasreddine ZS, Phillips NA, Bédirian V, Charbonneau S, Whitehead V, Collin I, et al. The Montreal Cognitive Assessment, MoCA: a brief screening tool for mild cognitive impairment. J Am Geriatr Soc 2005;53:695-9.

[36] Pantoni L, Basile AM, Pracucci G, Asplund K, Bogousslavsky J, Chabriat $\mathrm{H}$, et al. Impact of age-related cerebral white matter changes on the transition to disability - the LADIS study: rationale, design and methodology. Neuroepidemiology 2005;24:51-62.

[37] Scheltens P, Barkhof F, Leys D, Pruvo JP, Nauta JJ, Vermersch $P$, et al. A semiquantative rating scale for the assessment of signal hyperintensities on magnetic resonance imaging. J Neurol Sci 1993;114:7-12.

[38] Agostoni E, Fumagalli L, Santoro P, Ferrarese C. Migraine and stroke. Neurol Sci 2004;25:123-5.

[39] Welch KM, Levine SR. Migraine-related stroke in the context of the International Headache Society classification of head pain. Arch Neurol 1990;47:458-62. 